Article

\title{
An Interval-Valued Intuitionistic Fuzzy MADM Method Based on a New Similarity Measure
}

\section{Haiping Ren ${ }^{1, *}$ and Guofu Wang ${ }^{2}$}

1 School of Software, Jiangxi University of Science and Technology, Nanchang 330013, China

2 School of Mathematics and Statistics, Central South University, Changsha 410000, China; E-Mail: wangguofu117@csu.edu.com

* Author to whom correspondence should be addressed; E-Mail: chinarhp@mail.jxust.edu.cn; Tel./Fax: +86-0791-83858391.

Academic Editor: Willy Susilo

Received: 26 September 2015 / Accepted: 8 December 2015 / Published: 18 December 2015

\begin{abstract}
Similarity measure is one of the most important measures of interval-valued intuitionistic fuzzy (IVIF) sets. This article will put forward a new similarity measure, which considers the impacts of membership degree, nonmembership degree and median point of IVIF sets. For cases with partially known attribute weight information in multi-attribute decision-making (MADM) problems, a new weighting method is put forward by establishing the maximum similarity optimization model to solve the optimal weights. Further, a new decision-making method is developed on the basis of proposed similarity measure, and an applied example proves the effectiveness and feasibility of the proposed methods.
\end{abstract}

Keywords: similarity measure; interval-valued intuitionistic fuzzy set; multi-attribute decision making method; maximum similarity optimization model

\section{Introduction}

Multi-attribute decision-making (MADM) theory has been widely applied in many fields such as economics, management science and engineering [1-3]. Due to the complexity and uncertainty of objective things and the ambiguity of human thinking, the fuzzy set introduced by Zadeh [4] seems to be suitable for describing subjective decision information $[5,6]$. With the increasing complexity and uncertainty of the social economic environment, there often exist different hesitancy degrees or a certain degree of lack of knowledge in the decision-making process. Atanassov [7] proposed IF sets, 
which can be seen as an extension of the Zadeh's fuzzy set. Due to the complexity and uncertainty of objective things and the limitation of the knowledge of decision makers, membership and nonmembership degree are sometimes very difficult to express with crisp numbers, and interval numbers can effectively describe these situations. For this reason, Atanassov and Gargov [8] extended the IF sets to the IVIF sets in 1989. Through introducing the non-membership and hesitancy degrees, IF sets and IVIF sets are made more suitable for expressing the decision maker's satisfaction and/or dissatisfaction degrees than crisp numbers, fuzzy sets or linguistic variables [9]. There are many studies of MADM problems under IF or IVIF environments [10-14].

As the core problem of intuitionistic fuzzy set theory, similarity has been studied by many scholars, and has been applied to the fields such as pattern recognition, medical diagnosis, clustering analysis and MADM problems. Similarity measure is an important tool for measuring the degree of resemblance between two fuzzy sets. Many similarity measures of fuzzy sets are investigated in the literature. Li and Chen [15] firstly gave the definition of the similarity measure between IF sets, and they also proposed some similarity measures, and then first used them in pattern recognition. Li and Chen's similarity measure takes into account the medians of two intervals only, and thus counter-intuitive examples can easily be pointed out. Mitchell [16] proposed an improved similarity based on Li and Chen's similarity measure from a statistical viewpoint. Baccour et al. [17] summarized the existing similarity measures and pointed out that each above similarity measure has drawbacks. More recently, Hwang and Yang [18] provided a new construction for similarity measures for IF sets by defining lower, upper and middle fuzzy sets, and they found that the newly constructed similarity measures can improve most existing similarity measures.

As an extension of IF sets, IVIF sets are also receiving great attention and being applied in many fields [11-13]. Similarity measure is one of the most important measures of IVIF sets. Motivated by the concept of one paper [18], we have developed a new similarity measure between two IVIF sets. This paper also develops a new decision-making method based on the proposed similarity measure for the MADM problem in an IVIF environment with partially known attribute weight information.

The rest of this paper is organized as follows: In Section 2, we first briefly review the definition and similarity measure of IVIF sets. Section 3 constructs a new similarity measure for IFSs. In Section 4, based on the new similarity measure, an optimization model is constructed to solve the attribute weights, and then we develop a new decision making method for the MADM problem in which attribute values expressed by IVIF numbers. Finally, conclusions are given in Section 5.

\section{Preliminary Knowledge}

In what follows, some basic concepts of IF sets and IVIF sets are introduced to facilitate the discussion. Atanassov [7] proposed the concept of IF sets, which is defined as in Definition 1.

Definition 1. Let $X=\left\{x_{1}, x_{2}, \cdots, x_{n}\right\}$ be a finite universe of discourse, then

$$
U=\left\{<x_{j}, \mu_{U}\left(x_{j}\right), \mathrm{v}_{U}\left(x_{j}\right)>\mid x_{j} \in X\right\}
$$

is called an IF set over $X$, where $\mu_{U}\left(x_{j}\right)$ and $v_{U}\left(x_{j}\right)$ denote membership degree and nonmembership degree of each element $x_{j}$ belongs to $U$, and they satisfy $\mu_{U}\left(x_{j}\right) \in[0,1], v_{U}\left(x_{j}\right) \in[0,1]$ and $\mu_{U}\left(x_{j}\right)+v_{U}\left(x_{j}\right) \leq 1 . \pi_{U}\left(x_{j}\right)=1-\mu_{U}\left(x_{j}\right)-v_{U}\left(x_{j}\right)$ is called the hesitation degree of an element $x_{j}$ to 
$U$. Obviously, $0 \leq \pi_{U}\left(x_{j}\right) \leq 1$ for every $x_{j} \in X$. And if $\pi_{U}\left(x_{j}\right)=0$, then the IF set $U$ is reduced to a fuzzy set, i.e., $U=\left\{<\mu_{U}\left(x_{j}\right), 1-\mu_{U}\left(x_{j}\right)>\mid x_{j} \in X\right\}$.

In some situations, it is very difficult to use crisp numbers to express $\mu_{U}\left(x_{j}\right)$ and $v_{U}\left(x_{j}\right)$ precisely for the complexity and uncertainties of the objective things. However, we can use intervals to express them easily. Therefore, Atanassov and Gargov [8] extended IF sets to the IVIF sets, and gave the definition of the IVIF sets as in Definition 2.

Definition 2. Let $X=\left\{x_{1}, x_{2}, \cdots, x_{n}\right\}$ be a finite universe of discourse, then

$$
\tilde{U}=\left\{<x_{j}, \tilde{\mu}_{\tilde{U}}\left(x_{j}\right), \tilde{v}_{\tilde{U}}\left(x_{j}\right)>\mid x_{j} \in X\right\}
$$

is called an IVIF set over $X$, where $\tilde{\mu}_{\tilde{U}}\left(x_{j}\right)=\left[\mu_{\tilde{U}}^{-}\left(x_{j}\right), \mu_{\tilde{U}}^{+}\left(x_{j}\right)\right]$ and $\tilde{\mu}_{\tilde{U}}\left(x_{j}\right)=\left[\mu_{\tilde{U}}^{-}\left(x_{j}\right), \mu_{\tilde{U}}^{+}\left(x_{j}\right)\right]$ are membership degree and nonmembership degree of each element $x_{j}$ belongs to $U$, respectively. They satisfy $\mu_{\tilde{U}}^{+}\left(x_{j}\right)+v_{\tilde{U}}^{+}\left(x_{j}\right) \leq 1$. Each element of $\tilde{U},\left(i . e .,<x_{j}, \tilde{\mu}_{\tilde{U}}\left(x_{j}\right), \tilde{v}_{\tilde{U}}\left(x_{j}\right)>\right)$ is called an IVIF number or IVIF value [17]. $\tilde{\pi}_{\tilde{U}}\left(x_{j}\right)=\left[\pi_{\tilde{U}}^{-}\left(x_{j}\right), \pi_{\tilde{U}}^{+}\left(x_{j}\right)\right]$ is called the hesitation degree of an IVIF number $<\tilde{\mu}_{\tilde{U}}\left(x_{j}\right), \tilde{v}_{\tilde{U}}\left(x_{j}\right)>$, where $\pi_{\tilde{U}}^{-}\left(x_{j}\right)=1-\mu_{\tilde{U}}^{+}\left(x_{j}\right)-v_{\tilde{U}}^{+}\left(x_{j}\right)$ and $\pi_{\tilde{U}}^{+}\left(x_{j}\right)=1-\mu_{\tilde{U}}^{-}\left(x_{j}\right)-v_{\tilde{U}}^{-}\left(x_{j}\right)$, for all $x_{j} \in X$.

We briefly denote an IVIF number $\tilde{A}=<\tilde{\mu}_{\tilde{U}}\left(x_{j}\right), \tilde{\mathrm{v}}_{\tilde{U}}\left(x_{j}\right)>$ and its hesitation degree $\tilde{\pi}_{\tilde{U}}\left(x_{j}\right)$ by $\tilde{A}=<\tilde{\mu}_{\tilde{A}}, \tilde{v}_{\tilde{A}}>$ and $\tilde{\pi}_{\tilde{A}}$ respectively, where

$$
\begin{aligned}
& \tilde{\mu}_{\tilde{A}}=\left[\mu_{\tilde{A}}^{-}, \mu_{\tilde{A}}^{+}\right] \subset[0,1], \tilde{\mathrm{v}}_{\tilde{A}}=\left[\mathrm{v}_{\tilde{A}}^{-}, \mathrm{v}_{\tilde{A}}^{+}\right] \subset[0,1], \mu_{\tilde{A}}^{+}+\mathrm{v}_{\tilde{A}}^{+} \leq 1 \\
& \tilde{\pi}_{\tilde{A}}=\left[\pi_{\tilde{A}}^{-}, \pi_{\tilde{A}}^{+}\right] \subset[0,1], \pi_{\tilde{A}}^{-}=1-\mu_{\tilde{A}}^{+}-\tilde{\mathrm{v}}_{\tilde{A}}^{+}, \pi_{\tilde{A}}^{+}=1-\mu_{\tilde{A}}^{-}-\tilde{\mathrm{v}}_{\tilde{A}}^{-}
\end{aligned}
$$

Definition 3. Let $\tilde{A}_{i}=<\tilde{\mu}_{\tilde{A}_{i}}, \tilde{\mathrm{v}}_{\tilde{A}_{i}}>(i=1,2)$ be two any IVIF numbers, then

(i) If $\mu_{\tilde{A}_{1}}^{-} \leq \mu_{\tilde{A}_{2}}^{-}, \mu_{\tilde{A}_{1}}^{+} \leq \mu_{\tilde{A}_{2}}^{+}$and $v_{\tilde{A}_{1}}^{-} \geq v_{\tilde{A}_{2}}^{-}, v_{\tilde{A}_{1}}^{+} \geq v_{\tilde{A}_{2}}^{+}$, then $\tilde{A}_{1}$ is no larger than $\tilde{A}_{2}$, and noted by $\tilde{A}_{1} \leq \tilde{A}_{2}$

(ii) If $\tilde{A}_{1} \leq \tilde{A}_{2}$ and $\tilde{A}_{1} \geq \tilde{A}_{2}$, then $\tilde{A}_{1}$ is equal to $\tilde{A}_{2}$, and noted by $\tilde{A}_{1}=\tilde{A}_{2}$.

By Definition $3, \tilde{A}^{+}=<[1,1],[0,0]>$ is the largest IVIF number; $\tilde{A}^{-}=<[0,0],[1,1]>$ is the smallest IVIF number.

Definition 4. Let $\tilde{A}=\left\{<x_{i}, \tilde{\mu}_{\tilde{A}}\left(x_{i}\right), \tilde{\mathrm{v}}_{\tilde{A}}\left(x_{i}\right)>\mid x_{i} \in X\right\}$ and $\tilde{B}=\left\{<x_{i}, \tilde{\mu}_{\tilde{B}}\left(x_{i}\right), \tilde{\mathrm{v}}_{\tilde{B}}\left(x_{i}\right)>\mid x_{i} \in X\right\}$ be two IVIF sets, then the following operations can be founded in [8]:

(i) The complementary set of $\tilde{A}$ denoted by $\tilde{A}^{C}$, is $\tilde{A}^{C}=\left\{<x_{i}, \tilde{v}_{\tilde{A}}\left(x_{i}\right), \tilde{\mu}_{\tilde{A}}\left(x_{i}\right)>\mid x_{i} \in X\right\}$;

(ii) $\tilde{A} \subseteq \tilde{B}$ if and only if $\forall x_{i} \in X, \mu_{\tilde{A}}^{-} \leq \mu_{\tilde{B}}^{-}, \mu_{\tilde{A}}^{+} \leq \mu_{\tilde{B}}^{+}$and $v_{\tilde{A}}^{-} \geq v_{\tilde{B}}^{-}, \mathrm{v}_{\tilde{A}}^{+} \geq \mathrm{v}_{\tilde{B}}^{+}$;

(iii) $\tilde{A}=\tilde{B}$, if and only if $\tilde{A} \subseteq \tilde{B}$ and $\tilde{B} \subseteq \tilde{A}$.

Definition 5. Let $\tilde{A}=\left\{<x_{i}, \tilde{\mu}_{\tilde{A}}\left(x_{i}\right), \tilde{\mathrm{v}}_{\tilde{A}}\left(x_{i}\right)>\mid x_{i} \in X\right\}$ and $\tilde{B}=\left\{<x_{i}, \tilde{\mu}_{\tilde{B}}\left(x_{i}\right), \tilde{\mathrm{v}}_{\tilde{B}}\left(x_{i}\right)>\mid x_{i} \in X\right\}$ be two IVIF sets and the weight of $x_{i}$ is $w_{i}$. Then the weighted Hamming distance of $\tilde{A}$ and $\tilde{B}$ is defined as [19]: 


$$
\begin{array}{r}
d(\tilde{A}, \tilde{B})=\frac{1}{4 n} \sum_{j=1}^{n} w_{j}\left[\left|\mu_{A}^{-}\left(x_{j}\right)-\mu_{B}^{-}\left(x_{j}\right)\right|+\left|\mu_{A}^{+}\left(x_{j}\right)-\mu_{B}^{+}\left(x_{j}\right)\right|+\left|\mathrm{v}_{A}^{-}\left(x_{j}\right)-\mathrm{v}_{B}^{-}\left(x_{j}\right)\right|\right. \\
\left.+\left|\mathrm{v}_{A}^{+}\left(x_{j}\right)-\mathrm{v}_{B}^{+}\left(x_{j}\right)\right|+\left|\pi_{A}^{-}\left(x_{j}\right)-\pi_{B}^{-}\left(x_{j}\right)\right|+\left|\pi_{A}^{+}\left(x_{j}\right)-\pi_{B}^{+}\left(x_{j}\right)\right|\right]
\end{array}
$$

Definition 6. Let $\tilde{\alpha}_{1}=<\left[a_{1}, b_{1}\right],\left[c_{1}, d_{1}\right]>$ and $\tilde{\alpha}_{2}=<\left[a_{2}, b_{2}\right],\left[c_{2}, d_{2}\right]>$ be two IVIF numbers. Then Ref. [20] gave the operation laws of IVIF numbers as follows:

(i) $\tilde{\alpha}_{1} \otimes \tilde{\alpha}_{2}=<\left[a_{1} a_{2}, b_{1} b_{2}\right],\left[c_{1}+c_{2}-c_{1} c_{2}, d_{1}+d_{2}-d_{1} d_{2}\right]>$;

(ii) $\tilde{\alpha}_{1}^{\lambda}=<\left[a_{1}^{\lambda}, b_{1}^{\lambda}\right],\left[1-\left(1-c_{1}\right)^{\lambda}, 1-\left(1-d_{1}\right)^{\lambda}\right]>, \lambda>0$.

Definition 7. Let $\tilde{\alpha}_{i}(i=1,2, \ldots, n)$ be a collection of IVIF numbers, and an IVIF hybrid geometric (IIFHG) operator is a mapping IIFHG:IVIFNs ${ }^{n} \rightarrow I V I F N s$, which is defined as [20]:

$$
I I F H G_{\lambda, w}\left(\tilde{\alpha}_{1}, \tilde{\alpha}_{2}, \ldots, \tilde{\alpha}_{n}\right)=\dot{\tilde{\alpha}}_{\sigma(1)}^{w_{1}} \otimes \dot{\tilde{\alpha}}_{\sigma(2)}^{w_{2}} \otimes \cdots \otimes \dot{\tilde{\alpha}}_{\sigma(n)}^{w_{n}}
$$

where $\boldsymbol{w}=\left(w_{1}, w_{2}, \ldots, w_{n}\right)^{T}$ is the position weights of IIFHG operator, $w_{i} \in[0,1] \quad(i=1,2, \ldots, n)$ and $\sum_{i=1}^{n} w_{i}=1 ; \dot{\tilde{\alpha}}_{\sigma(i)}$ is the $i$ th largest of the weighted IVIF numbers. $\dot{\tilde{\alpha}}_{j}=\tilde{\alpha}_{j}^{n \lambda_{j}}(j=1,2, \ldots, n)$; $\lambda=\left(\lambda_{1}, \lambda_{2}, \ldots, \lambda_{n}\right)^{T}$ is the weight vector of IVIF numbers. $\tilde{\alpha}_{i}(i=1,2, \ldots, n)$ with $0 \leq \lambda_{j} \leq 1(j=1,2, \ldots, n)$ and $\sum_{j=1}^{n} \lambda_{j}=1$.

In the following discussion, we always suppose that $\operatorname{IVIFS} s(X)$ is the set of all IVIF sets defined in $X$.

Definition 8. Let $\tilde{A}=\left\{<x_{i}, \tilde{\mu}_{\tilde{A}}\left(x_{i}\right), \tilde{\mathrm{v}}_{\tilde{A}}\left(x_{i}\right)>\mid x_{i} \in X\right\}$ and $\tilde{B}=\left\{<x_{i}, \tilde{\mu}_{\tilde{B}}\left(x_{i}\right), \tilde{\mathrm{v}}_{\tilde{B}}\left(x_{i}\right)>\mid x_{i} \in X\right\}$ be two IVIF sets, and let $S$ be a mapping $S: \operatorname{IVIFS} s(X) \times \operatorname{IVIFS} s(X) \rightarrow[0,1]$. Then $S(A, B)$ is called the similarity measure between $A$ and $B$ if it satisfies the following conditions [21]:

(i) If $\tilde{A}=\tilde{B}$, then $S(\tilde{A}, \tilde{B})=1$

(ii) $S(\tilde{A}, \tilde{B})=S(\tilde{B}, \tilde{A})$

(iii) If $\tilde{A} \subseteq \tilde{B} \subseteq \tilde{C}$, then $S(\tilde{A}, \tilde{C}) \leq \min \{S(\tilde{A}, \tilde{B}), S(\tilde{B}, \tilde{C})\}$

(iv) If $\tilde{A}$ is a crisp set, then $S\left(\tilde{A}, \tilde{A}^{C}\right)=0$.

\section{A New Similarity of IVIF Sets}

The main task of this section is to put forward an effective IVIF similarity, which considers the impacts of membership degree, nonmembership degree and median point of IVIF sets. In the following, we first introduce an IVIF operator:

$$
F_{p}(A)=\left\{<x, \mu_{A}^{-}(x)+p \Delta \mu_{A}(x), \mathrm{v}_{A}^{-}(x)+p \Delta \mathrm{v}_{A}(x)>\mid x \in X\right\}
$$

where $\Delta \mu_{A}(x)=\mu_{A}^{+}(x)-\mu_{A}^{-}(x), \Delta \mathrm{v}_{A}(x)=\mathrm{v}_{A}^{+}(x)-\mathrm{v}_{A}^{-}(x)$ and $p \in[0,1]$ is a parameter called the attitude factor, which reflects the degree of interval. 
Set $\bar{\mu}_{A}(x)=\mu_{A}^{-}(x)+p \Delta \mu_{A}(x), \overline{\mathrm{v}}_{A}(x)=\mathrm{v}_{A}^{-}(x)+p \Delta \mathrm{v}_{A}(x)>$, for any $p \in[0,1]$, then the new similarity is defined as follows:

Definition 9. Let $A=\left\{\left(x_{i}, \tilde{\mu}_{A}\left(x_{i}\right), \tilde{\mathrm{v}}_{A}\left(x_{i}\right)\right) \mid x_{i} \in X\right\}$ and $B=\left\{\left(x_{i}, \tilde{\mu}_{B}\left(x_{i}\right), \tilde{\mathrm{v}}_{B}\left(x_{i}\right)\right) \mid x_{i} \in X\right\}$ are two IVIF sets, then we define a new information measure between $A$ and $B$ as follows:

$$
S_{R}(A, B)=1-\frac{1}{2 n} \sum_{i=1}^{n}\left[\frac{\left|\bar{\mu}_{A}\left(x_{i}\right)-\bar{\mu}_{B}\left(x_{i}\right)\right|}{\bar{\mu}_{A}\left(x_{i}\right)+\bar{\mu}_{B}\left(x_{i}\right)}+\frac{\left|\overline{\mathrm{v}}_{A}\left(x_{i}\right)-\overline{\mathrm{v}}_{B}\left(x_{i}\right)\right|}{3-\overline{\mathrm{v}}_{A}\left(x_{i}\right)-\overline{\mathrm{v}}_{B}\left(x_{i}\right)}+\left|\bar{m}_{A}\left(x_{i}\right)-\bar{m}_{B}\left(x_{i}\right)\right|\right]
$$

where $\bar{m}_{A}\left(x_{i}\right)=\frac{\bar{\mu}_{A}\left(x_{i}\right)+1-\bar{v}_{A}\left(x_{i}\right)}{2}$ and $\bar{m}_{B}\left(x_{i}\right)=\frac{\bar{\mu}_{B}\left(x_{i}\right)+1-\overline{\mathrm{v}}_{B}\left(x_{i}\right)}{2}$.

Theorem 1. The measure given by Equation (8) is an IVIF similarity.

Proof. For any $p \in[0,1]$, we have

$$
\begin{aligned}
& 0 \leq \bar{\mu}_{A}(x)=\mu_{A}^{-}(x)+p \Delta \mu_{A}(x)=(1-p) \mu_{A}^{-}(x)+p \mu_{A}^{+}(x) \leq 1 \\
& 0 \leq \bar{v}_{A}(x)=v_{A}^{-}(x)+p \Delta v_{A}(x)=(1-p) v_{A}^{-}(x)+p v_{A}^{+}(x) \leq 1
\end{aligned}
$$

Besides

$$
\begin{aligned}
& 0 \leq \bar{m}_{A}\left(x_{i}\right)=\frac{\bar{\mu}_{A}\left(x_{i}\right)+1-\overline{\mathrm{v}}_{A}\left(x_{i}\right)}{2} \leq 1, \\
& 0 \leq \bar{m}_{B}\left(x_{i}\right)=\frac{\bar{\mu}_{B}\left(x_{i}\right)+1-\overline{\mathrm{v}}_{B}\left(x_{i}\right)}{2} \leq 1
\end{aligned}
$$

Construct the following function:

$$
f(x, y)=\frac{|x-y|}{1+x+y}
$$

Then, if $0 \leq x \leq y \leq 1$, then

$$
f(x, y)=\frac{|x-y|}{1+x+y}=\frac{y-x}{1+x+y} \leq \frac{y}{1+y} \leq \frac{y}{y+y}=\frac{1}{2}
$$

Similarly, if $0 \leq y \leq x \leq 1$, then $f(x, y) \leq \frac{1}{2}$. Thus for any real numbers $x, y \in[0,1], f(x, y) \leq \frac{1}{2}$ always holds true.

Therefore, for any $x_{i} \in X$, we have

$$
\begin{aligned}
& \frac{\left|\bar{\mu}_{A}\left(x_{i}\right)-\bar{\mu}_{B}\left(x_{i}\right)\right|}{1+\bar{\mu}_{A}\left(x_{i}\right)+\bar{\mu}_{B}\left(x_{i}\right)}+\frac{\left|\overline{\mathrm{v}}_{A}\left(x_{i}\right)-\overline{\mathrm{v}}_{B}\left(x_{i}\right)\right|}{3-\overline{\mathrm{v}}_{A}\left(x_{i}\right)-\overline{\mathrm{v}}_{B}\left(x_{i}\right)}+\left|\bar{m}_{A}\left(x_{i}\right)-\bar{m}_{B}\left(x_{i}\right)\right| \\
& =f\left(\bar{\mu}_{A}\left(x_{i}\right), \bar{\mu}_{B}\left(x_{i}\right)\right)+f\left(1-\overline{\mathrm{v}}_{A}\left(x_{i}\right), 1-\overline{\mathrm{v}}_{B}\left(x_{i}\right)\right)+\left|\bar{m}_{A}\left(x_{i}\right)-\bar{m}_{B}\left(x_{i}\right)\right| \\
& \leq \frac{1}{2}+\frac{1}{2}+1 \\
& \leq 2
\end{aligned}
$$

Then $0 \leq S_{R}(A, B) \leq 1$.

(i) If $A=B$, then $\tilde{\mu}_{A}\left(x_{i}\right)=\tilde{\mu}_{B}\left(x_{i}\right), \tilde{\mathrm{v}}_{A}\left(x_{i}\right)=\tilde{\mathrm{v}}_{B}\left(x_{i}\right), \forall x_{i} \in X$,

That is for any $x_{i} \in X, \mu_{A}^{-}\left(x_{i}\right)=\mu_{B}^{-}\left(x_{i}\right), \mu_{A}^{+}\left(x_{i}\right)=\mu_{B}^{+}\left(x_{i}\right)$ and $\mathrm{v}_{A}^{-}\left(x_{i}\right)=\mathrm{v}_{B}^{-}\left(x_{i}\right), \mathrm{v}_{A}^{+}\left(x_{i}\right)=\mathrm{v}_{B}^{+}\left(x_{i}\right)$;

Then for any $x_{i} \in X, \bar{\mu}_{A}\left(x_{i}\right)=\bar{\mu}_{B}\left(x_{i}\right), \bar{v}_{A}\left(x_{i}\right)=\bar{v}_{B}\left(x_{i}\right), \bar{m}_{A}\left(x_{i}\right)=\bar{m}_{B}\left(x_{i}\right)$, i.e., $S_{R}(A, B)=1$. 
(ii)

$$
\begin{aligned}
S_{R}(B, A) & =1-\frac{1}{2 n} \sum_{i=1}^{n}\left[\frac{\left|\bar{\mu}_{B}\left(x_{i}\right)-\bar{\mu}_{A}\left(x_{i}\right)\right|}{1+\bar{\mu}_{B}\left(x_{i}\right)+\bar{\mu}_{A}\left(x_{i}\right)}+\frac{\left|\overline{\mathrm{v}}_{B}\left(x_{i}\right)-\overline{\mathrm{v}}_{A}\left(x_{i}\right)\right|}{3-\overline{\mathrm{v}}_{B}\left(x_{i}\right)-\overline{\mathrm{v}}_{A}\left(x_{i}\right)}+\left|\bar{m}_{B}\left(x_{i}\right)-\bar{m}_{A}\left(x_{i}\right)\right|\right] \\
& =1-\frac{1}{2 n} \sum_{i=1}^{n}\left[\frac{\left|\bar{\mu}_{A}\left(x_{i}\right)-\bar{\mu}_{B}\left(x_{i}\right)\right|}{1+\bar{\mu}_{A}\left(x_{i}\right)+\bar{\mu}_{B}\left(x_{i}\right)}+\frac{\left|\overline{\mathrm{v}}_{A}\left(x_{i}\right)-\overline{\mathrm{v}}_{B}\left(x_{i}\right)\right|}{3-\overline{\mathrm{v}}_{A}\left(x_{i}\right)-\overline{\mathrm{v}}_{B}\left(x_{i}\right)}+\left|\bar{m}_{A}\left(x_{i}\right)-\bar{m}_{B}\left(x_{i}\right)\right|\right]=S_{R}(A, B)
\end{aligned}
$$

(iii) Let $f(x, y)=\frac{|x-y|}{1+x+y}$, then for any $0 \leq a \leq b \leq c$, we have

$$
\begin{aligned}
& f(a, c)-f(a, b) \\
& =\frac{|a-c|}{1+a+c}-\frac{|a-b|}{1+a+b}=\frac{(c-a)(1+a+b)-(b-a)(1+a+c)}{(1+a+c)(1+a+b)} \\
& =\frac{c-a+a c+b c-a^{2}-a b-b+a-a b-b c+a^{2}+a c}{(1+a+c)(1+a+b)} \\
& =\frac{(1+2 a)(c-b)}{(1+a+c)(1+a+b)} \geq 0 \\
& f(a, c)-f(b, c) \\
& =\frac{|a-c|}{1+a+c}-\frac{|b-c|}{1+b+c}=\frac{(c-a)(1+b+c)-(c-b)(1+a+c)}{(1+a+c)(1+b+c)} \\
& =\frac{c-a+b c+c^{2}-a b-a c-c+b-a c-c^{2}+a b+b c}{(1+a+c)(1+a+b)} \\
& =\frac{(1+2 c)(b-a)}{(1+a+c)(1+a+b)} \geq 0
\end{aligned}
$$

That is for any $0 \leq a \leq b \leq c$, we have $f(a, c) \geq f(a, b)$ and $f(a, c) \geq f(b, c)$.

If $A \subseteq B \subseteq C$, then for any $x_{i} \in X$, then

$$
\begin{aligned}
& \mu_{A}^{-}\left(x_{i}\right) \leq \mu_{B}^{-}\left(x_{i}\right) \leq \mu_{C}^{-}\left(x_{i}\right), \mu_{A}^{+}\left(x_{i}\right) \leq \mu_{B}^{+}\left(x_{i}\right) \leq \mu_{C}^{+}\left(x_{i}\right), \\
& \mathrm{v}_{A}^{-}\left(x_{i}\right) \geq \mathrm{v}_{B}^{-}\left(x_{i}\right) \geq \mathrm{v}_{C}^{-}\left(x_{i}\right), \mathrm{v}_{A}^{+}\left(x_{i}\right) \geq \mathrm{v}_{B}^{+}\left(x_{i}\right) \geq \mathrm{v}_{C}^{+}\left(x_{i}\right)
\end{aligned}
$$

Then

$$
\begin{aligned}
& \bar{\mu}_{A}(x)=(1-p) \mu_{A}^{-}(x)+p \mu_{A}^{+}(x) \leq(1-p) \mu_{B}^{-}(x)+p \mu_{B}^{+}(x)=\bar{\mu}_{B}(x), \\
& \bar{v}_{A}(x)=(1-p) v_{A}^{-}(x)+p v_{A}^{+}(x) \geq(1-p) v_{B}^{-}(x)+p v_{B}^{+}(x)=\bar{v}_{B}(x)
\end{aligned}
$$

Similarly, we can get the following result:

$$
\bar{\mu}_{A}(x) \leq \bar{\mu}_{B}(x) \leq \bar{\mu}_{C}(x) \text { and } \overline{\mathrm{v}}_{A}(x) \geq \overline{\mathrm{v}}_{B}(x) \geq \overline{\mathrm{v}}_{C}(x)
$$

Consequently

$$
\begin{aligned}
& f\left(\bar{\mu}_{A}\left(x_{i}\right), \bar{\mu}_{C}\left(x_{i}\right)\right) \geq f\left(\bar{\mu}_{A}\left(x_{i}\right), \bar{\mu}_{B}\left(x_{i}\right)\right), \\
& f\left(\bar{\mu}_{A}\left(x_{i}\right), \bar{\mu}_{C}\left(x_{i}\right)\right) \geq f\left(\bar{\mu}_{B}\left(x_{i}\right), \bar{\mu}_{C}\left(x_{i}\right)\right), \\
& f\left(1-\overline{\mathrm{v}}_{A}\left(x_{i}\right), 1-\overline{\mathrm{v}}_{C}\left(x_{i}\right)\right) \geq f\left(1-\overline{\mathrm{v}}_{A}\left(x_{i}\right), 1-\overline{\mathrm{v}}_{B}\left(x_{i}\right)\right), \\
& f\left(1-\overline{\mathrm{v}}_{A}\left(x_{i}\right), 1-\overline{\mathrm{v}}_{C}\left(x_{i}\right)\right) \geq f\left(1-\overline{\mathrm{v}}_{B}\left(x_{i}\right), 1-\overline{\mathrm{v}}_{C}\left(x_{i}\right)\right)
\end{aligned}
$$


Obviously, $\bar{m}_{A}\left(x_{i}\right) \leq \bar{m}_{B}\left(x_{i}\right) \leq \bar{m}_{C}\left(x_{i}\right)$, and then we can get

$$
\left|\bar{m}_{A}\left(x_{i}\right)-\bar{m}_{C}\left(x_{i}\right)\right| \geq\left|\bar{m}_{A}\left(x_{i}\right)-\bar{m}_{B}\left(x_{i}\right)\right|,\left|\bar{m}_{A}\left(x_{i}\right)-\bar{m}_{C}\left(x_{i}\right)\right| \geq\left|\bar{m}_{B}\left(x_{i}\right)-\bar{m}_{C}\left(x_{i}\right)\right|
$$

Then

$$
\begin{aligned}
& f\left(\bar{\mu}_{A}\left(x_{i}\right), \bar{\mu}_{C}\left(x_{i}\right)\right)+f\left(1-\overline{\mathrm{v}}_{A}\left(x_{i}\right), 1-\overline{\mathrm{v}}_{C}\left(x_{i}\right)\right)+\left|\bar{m}_{A}\left(x_{i}\right)-\bar{m}_{C}\left(x_{i}\right)\right| \\
& \geq f\left(\bar{\mu}_{A}\left(x_{i}\right), \bar{\mu}_{B}\left(x_{i}\right)\right)+f\left(1-\overline{\mathrm{v}}_{A}\left(x_{i}\right), 1-\overline{\mathrm{v}}_{B}\left(x_{i}\right)\right)+\left|\bar{m}_{A}\left(x_{i}\right)-\bar{m}_{B}\left(x_{i}\right)\right|,
\end{aligned}
$$

and

$$
\begin{aligned}
& f\left(\bar{\mu}_{A}\left(x_{i}\right), \bar{\mu}_{C}\left(x_{i}\right)\right)+f\left(1-\overline{\mathrm{v}}_{A}\left(x_{i}\right), 1-\overline{\mathrm{v}}_{C}\left(x_{i}\right)\right)+\left|\bar{m}_{A}\left(x_{i}\right)-\bar{m}_{C}\left(x_{i}\right)\right| \\
& \geq f\left(\bar{\mu}_{B}\left(x_{i}\right), \bar{\mu}_{C}\left(x_{i}\right)\right)+f\left(1-\overline{\mathrm{v}}_{B}\left(x_{i}\right), 1-\overline{\mathrm{v}}_{C}\left(x_{i}\right)\right)+\left|\bar{m}_{B}\left(x_{i}\right)-\bar{m}_{C}\left(x_{i}\right)\right|
\end{aligned}
$$

Finally, it holds true that

$$
\begin{aligned}
& S_{R}(A, C)=1-\frac{1}{2 n} \sum_{i=1}^{n}\left[f\left(\bar{\mu}_{A}\left(x_{i}\right), \bar{\mu}_{C}\left(x_{i}\right)\right)+f\left(1-\overline{\mathrm{v}}_{A}\left(x_{i}\right), 1-\overline{\mathrm{v}}_{C}\left(x_{i}\right)\right)+\left|\bar{m}_{A}\left(x_{i}\right)-\bar{m}_{C}\left(x_{i}\right)\right|\right] \\
& \leq 1-\frac{1}{2 n} \sum_{i=1}^{n}\left[f\left(\bar{\mu}_{A}\left(x_{i}\right), \bar{\mu}_{B}\left(x_{i}\right)\right)+f\left(1-\overline{\mathrm{v}}_{A}\left(x_{i}\right), 1-\overline{\mathrm{v}}_{B}\left(x_{i}\right)\right)+\left|\bar{m}_{A}\left(x_{i}\right)-\bar{m}_{B}\left(x_{i}\right)\right|\right]=S_{R}(A, B) \\
& S_{R}(A, C)=1-\frac{1}{2 n} \sum_{i=1}^{n}\left[f\left(\bar{\mu}_{A}\left(x_{i}\right), \bar{\mu}_{C}\left(x_{i}\right)\right)+f\left(1-\overline{\mathrm{v}}_{A}\left(x_{i}\right), 1-\overline{\mathrm{v}}_{C}\left(x_{i}\right)\right)+\left|\bar{m}_{A}\left(x_{i}\right)-\bar{m}_{C}\left(x_{i}\right)\right|\right] \\
& \leq 1-\frac{1}{2 n} \sum_{i=1}^{n}\left[f\left(\bar{\mu}_{B}\left(x_{i}\right), \bar{\mu}_{C}\left(x_{i}\right)\right)+f\left(1-\overline{\mathrm{v}}_{B}\left(x_{i}\right), 1-\overline{\mathrm{v}}_{C}\left(x_{i}\right)\right)+\left|\bar{m}_{B}\left(x_{i}\right)-\bar{m}_{C}\left(x_{i}\right)\right|\right]=S_{R}(B, C)
\end{aligned}
$$

(iv) When $A$ is a crisp set, that is

$$
\left[\mu_{A}^{-}\left(x_{i}\right), \mu_{A}^{+}\left(x_{i}\right)\right]=[0,0] \text { or }[1,1] \text {, and } \mathrm{v}_{A}^{-}\left(x_{i}\right)=1-\mu_{A}^{-}\left(x_{i}\right), \mathrm{v}_{A}^{+}\left(x_{i}\right)=1-\mu_{A}^{+}\left(x_{i}\right) .
$$

Thus for any $x_{i} \in X$,

(a) If $\left[\mu_{A}^{-}\left(x_{i}\right), \mu_{A}^{+}\left(x_{i}\right)\right]=[0,0]$, then

$$
\begin{gathered}
\bar{\mu}_{A}(x)=(1-p) \mu_{A}^{-}(x)+p \mu_{A}^{+}(x)=0 \\
\bar{v}_{A}(x)=(1-p) v_{A}^{-}(x)+p v_{A}^{+}(x)=1 \\
\bar{m}_{A}\left(x_{i}\right)=\frac{\bar{\mu}_{A}\left(x_{i}\right)+1-\bar{v}_{A}\left(x_{i}\right)}{2}=0
\end{gathered}
$$

Thus

$$
S_{R}\left(A, A^{C}\right)=1-\frac{1}{2 n} \sum_{i=1}^{n}\left[\frac{|0-1|}{1+0+1}+\frac{|1-0|}{3-1-0}+|0-1|\right]=0
$$

(b) Case (b) similar to case (a) considering $\left[\mu_{A}^{-}\left(x_{i}\right), \mu_{A}^{+}\left(x_{i}\right)\right]=[1,1]$, we can also prove the result $S_{R}\left(A, A^{C}\right)=0$.

In many situations, the important degree of the element $x_{i} \in X$ should be taken into account; for example, in MADM, the considered attributes usually have different levels of importance, and thus need to be assigned different weights. On the basis of Equation (8), we further define a weighted similarity measure between two IVIF sets $A$ and $B$ as follows: 


$$
S_{W R}(A, B)=1-\frac{1}{2} \sum_{i=1}^{n} w_{i}\left[\frac{\left|\bar{\mu}_{A}\left(x_{i}\right)-\bar{\mu}_{B}\left(x_{i}\right)\right|}{1+\bar{\mu}_{A}\left(x_{i}\right)+\bar{\mu}_{B}\left(x_{i}\right)}+\frac{\left|\overline{\mathrm{v}}_{A}\left(x_{i}\right)-\overline{\mathrm{v}}_{B}\left(x_{i}\right)\right|}{3-\overline{\mathrm{v}}_{A}\left(x_{i}\right)-\overline{\mathrm{v}}_{B}\left(x_{i}\right)}+\left|\bar{m}_{A}\left(x_{i}\right)-\bar{m}_{B}\left(x_{i}\right)\right|\right]
$$

where $w_{i} \in[0,1](i=1,2, \ldots, n)$ is the important degree of $x_{i}$, and $\sum_{i=1}^{n} w_{i}=1$.

If we set $w_{i}=1 / n \quad(i=1,2, \ldots, n)$, then $S_{W R}(A, B)=S_{R}(A, B)$. Similar to the proof process of $S_{R}(A, B)$, we can easily prove that the weighted similarity measure $S_{W R}(A, B)$ also satisfies the conditions in Definition 8.

\section{A New MADM Method Based on the Proposed Similarity}

\subsection{Description of the MADM Problem}

An MADM method consists on finding the best alternative from a set $A=\left\{A_{1}, A_{2}, \ldots, A_{m}\right\}$ of $m$ alternatives with respect to a set $O=\left\{o_{1}, o_{2}, \cdots, o_{n}\right\}$ of $n$ attributes. Suppose that the ratings of alternatives $A_{i} \in A$ on attributes $o_{j} \in O$ are expressed by IVIF number $\tilde{a}_{i j}=<\left[\mu_{i j}^{-}, \mu_{i j}^{+}\right],\left[\mathrm{v}_{i j}^{-}, \mathrm{v}_{i j}^{+}\right]>$, where $\left[\mu_{i j}^{-}, \mu_{i j}^{+}\right]$and $\left[v_{i j}^{-}, v_{i j}^{+}\right]$are intervals, which express the membership (satisfactory) and nonmembership (nonsatisfactory) degree of the alternative $A_{i} \in A$ on the attribute $o_{j} \in O$ with respect to the fuzzy concept "excellence" given by the decision maker so that they satisfy the conditions: $\mu_{i j}^{-}, \mu_{i j}^{+}, v_{i j}^{-}, v_{i j}^{+} \in[0,1]$ and $0 \leq \mu_{i j}^{+}+v_{i j}^{+} \leq 1(i=1,2, \cdots, m ; j=1,2, \cdots, n)$.

Thus, an MADM problem can be expressed with the following decision matrix:

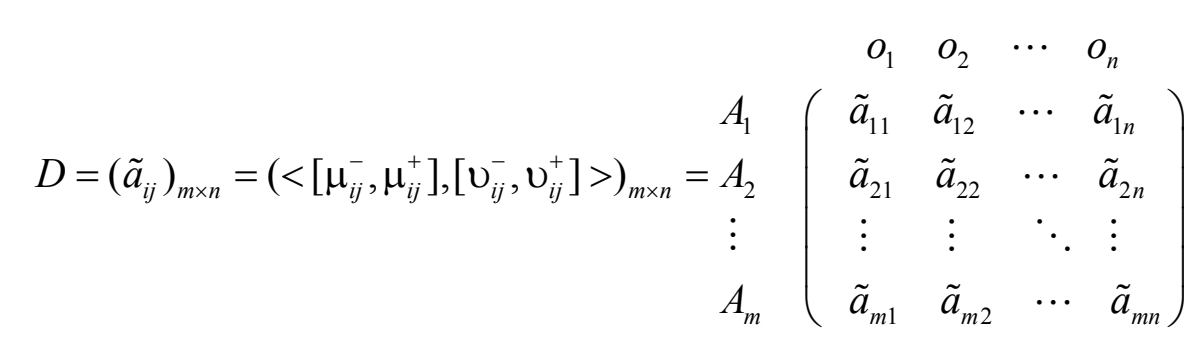

Let $\boldsymbol{W}=\left(w_{1}, w_{2}, \ldots, w_{n}\right)^{T}$ be the weight vector of all attributes, where $0 \leq w_{j} \leq 1(j=1,2, \cdots, m)$ is weight of each attribute $o_{j} \in O$, and $\sum_{j=1}^{n} w_{j}=1$. The attribute weight information is usually unknown or partially known due to the insufficient knowledge or limitation of time of decision makers in the decision-making process. In this paper, we will put forward a new weighting method to determine the attribute weights when only partial information about the weights is known.

\subsection{Weight-Determining Method}

In real decision situations, due to the complexity and uncertainty of practical decision making problems and the inherent subjective nature of human thinking, the attribute weight information is usually incomplete [22-26]. Generally, there will have more constraint conditions for weight vector $\boldsymbol{W}=\left(w_{1}, w_{2}, \ldots, w_{n}\right)^{T}$. We denote $\boldsymbol{H}$ as the set of the known weight information.

To determine the attribute weights for MADM problem with partially known attribute weight information in an intuitionistic fuzzy environment, $\mathrm{Xu}$ [22] proposed an optimization model based on 
Chen and Tan's score function [23]; Wu and Zhang [24], Wang and Wang [25] determined the attribute weights by establishing a programming model according to the minimum entropy principle. In this paper, we will use the new similarity measure to determine the attribute weights, and the method is similar to that of Chen and Yang [26]. Then, for the MADM problem $\tilde{D}=\left(\tilde{a}_{i j}\right)_{n \times m}$ with incomplete attribute weight information $\boldsymbol{W} \in \boldsymbol{H}$, the weight determining method is given as follows:

Step 1. Define the positive ideal solution (PIS) $A^{+}$of the IVIF MADM problem as follows: $A^{+}=\left(\tilde{a}_{1}^{+}, \tilde{a}_{2}^{+}, \cdots, \tilde{a}_{n}^{+}\right)$, where $\tilde{a}_{j}^{+}=<[1,1],[0,0]>(j=1,2, \cdots, n)$.

Step 2. Calculate weighted similarity between alternative $A_{i}=\left(\tilde{a}_{i 1}, \tilde{a}_{i 2}, \ldots, \tilde{a}_{i n}\right)$ with PIS ( $\left.A^{+}\right)$ as follows:

According to Equation (9), for $i=1,2, \ldots, m$,

$$
\begin{gathered}
S\left(A_{i}\right)=1-\frac{1}{2} \sum_{j=1}^{n} w_{j}\left[\frac{1-(1-p) \mu_{i j}^{-}-p \mu_{i j}^{+}}{2+(1-p) \mu_{i j}^{-}+p \mu_{i j}^{+}}+\frac{(1-p) v_{i j}^{-}+p v_{i j}^{+}}{3-(1-p) v_{i j}^{-}-p v_{i j}^{+}}\right. \\
\left.+1-\frac{(1-p)\left(\mu_{i j}^{-}+v_{i j}^{-}\right)+1-p\left(\mu_{i j}^{+}+v_{i j}^{+}\right)}{2}\right]
\end{gathered}
$$

Remark 1. To rank the alternatives according to the decision matrix $D=\left(\tilde{a}_{i j}\right)_{m \times n}$, the optimal weights of attributes should give the similarity between each alternative with PIS for the maximum. Then we can establish the following programming:

For $i=1,2, \ldots, m$,

$$
\begin{aligned}
& \max S\left(A_{i}\right) \\
& \text { s.t. }\left\{\begin{array}{l}
\sum_{j=1}^{n} w_{j}=1 \\
\boldsymbol{w} \in H
\end{array}\right.
\end{aligned}
$$

Here, $\boldsymbol{H}$ is the set of the known weight information.

Because each alternative is fair competition, the weight vector should make the similarities of all alternatives with PIS for the maximum, that is

$$
\begin{aligned}
\max S & =\sum_{i=1}^{m} S\left(A_{i}\right) \\
& =m-\frac{1}{2} \sum_{i=1}^{m} \sum_{j=1}^{n} w_{j}\left[\frac{1-(1-p) \mu_{i j}^{-}-p \mu_{i j}^{+}}{2+(1-p) \mu_{i j}^{-}+p \mu_{i j}^{+}}+\frac{(1-p) \mathrm{v}_{i j}^{-}+p v_{i j}^{+}}{3-(1-p) \mathrm{v}_{i j}^{-}-p v_{i j}^{+}}+1-\frac{(1-p)\left(\mu_{i j}^{-}+v_{i j}^{-}\right)+1-p\left(\mu_{i j}^{+}+v_{i j}^{+}\right)}{2}\right]
\end{aligned}
$$

Then we can establish the following optimization model: 


$$
\begin{gathered}
\max S=m-\frac{1}{2} \sum_{i=1}^{m} \sum_{j=1}^{n} w_{j}\left[\frac{1-(1-p) \mu_{i j}^{-}-p \mu_{i j}^{+}}{2+(1-p) \mu_{i j}^{-}+p \mu_{i j}^{+}}+\frac{(1-p) v_{i j}^{-}+p v_{i j}^{+}}{3-(1-p) v_{i j}^{-}-p v_{i j}^{+}}\right. \\
\left.\qquad 1-\frac{(1-p)\left(\mu_{i j}^{-}+v_{i j}^{-}\right)+1-p\left(\mu_{i j}^{+}+v_{i j}^{+}\right)}{2}\right] \\
\text { s.t. }\left\{\begin{array}{l}
\sum_{j=1}^{n} w_{j}=1 \\
\boldsymbol{w} \in H
\end{array}\right.
\end{gathered}
$$

Step 3. Solve the Equation (14), then the optimal solution $\boldsymbol{w}^{*}=\arg \min E$ is chosen as the optimal attribute weights.

\subsection{New MADM Method Based on the Proposed Similarity}

In this subsection, we put forward the new MADM method based on the above-mentioned work. The specific calculation steps are given as follows:

Step 1. Establish the MADM model with IVIF information decision matrix;

Step 2. Calculate the attribute weights according to Equation (14);

Step 3. Determine the positive ideal solution (PIS) of the IVIF MADM problem as follows:

The PIS is defined as $A^{*}=\left(\tilde{a}_{1}^{*}, \tilde{a}_{2}^{*}, \cdots, \tilde{a}_{n}^{*}\right)$, where $\tilde{a}_{j}^{*}=<[1,1],[0,0]>(j=1,2, \cdots, n)$.

Step 4. Calculate the similarity measure $S\left(A_{i}\right)$ between alternative $A_{i}$ with PIS according to the weighted similarity measure defined in Equation (11).

Step 5. Rank the alternatives according to the similarity measures $S\left(A_{i}\right)$. The larger the value of $S\left(A_{i}\right)$ the better the alternative $A_{i}$. When $S\left(A_{i}\right)=S\left(A_{j}\right)(i \neq j)$, we need adopt other ranking method, such as TOPSIS method or VIKOR method for further judgement.

\section{Numerical Example}

In order to illustrate the effectiveness and practicability of the proposed MADM method, an example of a risk investment decision-making problem adopted from Park et al. [27] is analyzed.

Suppose that a manufacturing company wants to search for the best global supplier for one of its most critical parts used in assembling process. The company hires four experts (decision makers) $D_{1}, D_{2}, D_{3}, D_{4}$ to evaluate four candidate suppliers: $A_{i}(i=1,2,3,4)$. The evaluation attributes are $o_{j}(j=1,2, \ldots, 5)$, which are defined as follows: overall cost of the product $\left(o_{1}\right)$, quality of the product $\left(o_{2}\right)$, service performance of supplier $\left(o_{3}\right)$, supplier's profile $\left(o_{4}\right)$, and risk factor $\left(o_{5}\right)$. After the expert discussion, get the evaluation values of each alternative on the different attributes as given by IVIF numbers, as shown in Tables $1-4$. The weight vector of the four experts is $\lambda=(0.3,0.2,0.3,0.2)^{T}$, and the corresponding position weight vector of IIFHG operator is $\boldsymbol{\omega}=(0.155,0.345,0.345,0.155)^{T}$. Now assume that the information of attribute weights is partially known, and the attribute weights satisfy

$$
\begin{gathered}
\boldsymbol{H}=\left\{w_{1} \leq 0.3,0.1 \leq w_{2} \leq 0.2,0.2 \leq w_{3} \leq 0.5,0.1 \leq w_{4} \leq 0.3, w_{5} \leq 0.4,\right. \\
\left.w_{3}-w_{2} \geq w_{5}-w_{4}, w_{4} \geq w_{1}, w_{3}-w_{1} \leq 0.1\right\}
\end{gathered}
$$


Table 1. IVIF information decision matrix for $D_{1}$.

\begin{tabular}{cccccc}
\hline \multirow{2}{*}{ Alternatives } & \multicolumn{5}{c}{ Attributes } \\
\cline { 2 - 6 } & $O_{1}$ & $O_{2}$ & $o_{3}$ & $o_{4}$ & $O_{5}$ \\
\hline \multirow{2}{*}{$A_{1}$} & $<[0.5,0.6]$, & $<[0.3,0.5]$, & $<[0.6,0.7]$, & $<[0.5,0.7]$, & $<[0.1,0.4]$, \\
& {$[0.2,0.3]>$} & {$[0.4,0.5]>$} & {$[0.2,0.3]>$} & {$[0.1,0.2]>$} & {$[0.3,0.5]>$} \\
\hline \multirow{2}{*}{$A_{2}$} & $<[0.3,0.4]$, & $<[0.1,0.3]$, & $<[0.3,0.4]$, & $<[0.2,0.4]$, & $<[0.7,0.8]$, \\
& {$[0.4,0.6]>$} & {$[0.2,0.4]>$} & {$[0.4,0.5]>$} & {$[0.5,0.6]>$} & {$[0.1,0.2]>$} \\
\hline \multirow{2}{*}{$A_{3}$} & $<[0.4,0.5]$, & $<[0.7,0.8]$, & $<[0.5,0.8]$, & $<[0.4,0.6]$, & $<[0.5,0.6]$, \\
& {$[0.3,0.5]>$} & {$[0.1,0.2]>$} & {$[0.1,0.2]>$} & {$[0.2,0.3]>$} & {$[0.2,0.3]>$} \\
\hline \multirow{2}{*}{$A_{4}$} & $<[0.3,0.5]$, & $<[0.1,0.2]$, & $<[0.1,0.2]$, & $<[0.2,0.3]$, & $<[0.2,0.3]$, \\
& {$[0.4,0.5]>$} & {$[0.7,0.8]>$} & {$[0.5,0.8]>$} & {$[0.4,0.6]>$} & {$[0.5,0.6]>$} \\
\hline
\end{tabular}

Table 2. IVIF information decision matrix for $D_{2}$.

\begin{tabular}{cccccc}
\hline \multirow{2}{*}{ Alternatives } & \multicolumn{6}{c}{ Attributes } \\
\cline { 2 - 6 } & $o_{1}$ & $o_{2}$ & $o_{3}$ & $o_{4}$ & $o_{5}$ \\
\hline \multirow{2}{*}{$A_{1}$} & $<[0.4,0.5]$, & $<[0.3,0.4]$, & $<[0.6,0.7]$, & $<[0.5,0.6]$, & $<[0.1,0.3]$, \\
& {$[0.2,0.4]>$} & {$[0.4,0.6]>$} & {$[0.1,0.2]>$} & {$[0.1,0.3]>$} & {$[0.3,0.5]>$} \\
\hline \multirow{2}{*}{$A_{2}$} & $<[0.3,0.5]$, & $<[0.1,0.3]$, & $<[0.3,0.4]$, & $<[0.2,0.3]$, & $<[0.6,0.8]$, \\
& {$[0.4,0.5]>$} & {$[0.3,0.7]>$} & {$[0.4,0.5]>$} & {$[0.6,0.7]>$} & {$[0.1,0.2]>$} \\
\hline \multirow{2}{*}{$A_{3}$} & $<[0.4,0.6]$, & $<[0.6,0.8]$, & $<[0.7,0.8]$, & $<[0.4,0.6]$, & $<[0.5,0.6]$, \\
& {$[0.3,0.4]>$} & {$[0.1,0.2]>$} & {$[0.1,0.2]>$} & {$[0.3,0.4]>$} & {$[0.2,0.4]>$} \\
\hline \multirow{2}{*}{$A_{4}$} & $<[0.3,0.4]$, & $<[0.1,0.2]$, & $<[0.1,0.2]$, & $<[0.3,0.4]$, & $<[0.2,0.4]$, \\
& {$[0.4,0.6]>$} & {$[0.6,0.8]>$} & {$[0.7,0.8]>$} & {$[0.4,0.6]>$} & {$[0.5,0.6]>$} \\
\hline
\end{tabular}

Table 3. IVIF information decision matrix for $D_{3}$.

\begin{tabular}{cccccc}
\hline \multirow{2}{*}{ Alternatives } & \multicolumn{5}{c}{ Attributes } \\
\cline { 2 - 6 } & $O_{1}$ & $O_{2}$ & $O_{3}$ & $o_{4}$ & $O_{5}$ \\
\hline \multirow{2}{*}{$A_{1}$} & $<[0.4,0.7]$, & $<[0.3,0.5]$, & $<[0.6,0.7]$, & $<[0.5,0.6]$, & $<[0.3,0.5]$, \\
& {$[0.1,0.2]>$} & {$[0.3,0.4]>$} & {$[0.1,0.2]>$} & {$[0.1,0.3]>$} & {$[0.4,0.5]>$} \\
\hline \multirow{2}{*}{$A_{2}$} & $<[0.4,0.5]$, & $<[0.2,0.4]$, & $<[0.4,0.5]$, & $<[0.1,0.2]$, & $<[0.6,0.7]$, \\
& {$[0.2,0.4]>$} & {$[0.4,0.5]>$} & {$[0.3,0.4]>$} & {$[0.7,0.8]>$} & {$[0.2,0.3]>$} \\
\hline \multirow{2}{*}{$A_{3}$} & $<[0.2,0.4]$, & $<[0.6,0.8]$, & $<[0.5,0.7]$, & $<[0.5,0.7]$, & $<[0.6,0.8]$, \\
& {$[0.3,0.4]>$} & {$[0.1,0.2]>$} & {$[0.1,0.3]>$} & {$[0.2,0.3]>$} & {$[0.1,0.2]>$} \\
\hline \multirow{2}{*}{$A_{4}$} & $<[0.3,0.4]$, & $<[0.1,0.2]$, & $<[0.1,0.3]$, & $<[0.2,0.3]$, & $<[0.1,0.2]$, \\
& {$[0.2,0.4]>$} & {$[0.6,0.8]>$} & {$[0.5,0.7]>$} & {$[0.5,0.7]>$} & {$[0.6,0.8]>$} \\
\hline
\end{tabular}


Table 4. IVIF information decision matrix for $D_{4}$.

\begin{tabular}{cccccc}
\hline \multirow{2}{*}{ Alternatives } & \multicolumn{5}{c}{ Attributes } \\
\cline { 2 - 6 } & $O_{1}$ & $O_{2}$ & $o_{3}$ & $o_{4}$ & $o_{5}$ \\
\hline \multirow{2}{*}{$A_{1}$} & $<[0.6,0.7]$, & $<[0.3,0.4]$, & $<[0.7,0.8]$, & $<[0.5,0.6]$, & $<[0.1,0.2]$, \\
& {$[0.2,0.3]>$} & {$[0.3,0.4]>$} & {$[0.1,0.2]>$} & {$[0.1,0.3]>$} & {$[0.5,0.7]>$} \\
\hline \multirow{2}{*}{$A_{2}$} & $<[0.4,0.5]$, & $<[0.1,0.2]$, & $<[0.3,0.4]$, & $<[0.2,0.3]$, & $<[0.6,0.7]$, \\
& {$[0.4,0.5]>$} & {$[0.2,0.3]>$} & {$[0.5,0.6]>$} & {$[0.4,0.6]>$} & {$[0.1,0.2]>$} \\
\hline \multirow{2}{*}{$A_{3}$} & $<[0.4,0.5]$, & $<[0.6,0.7]$, & $<[0.5,0.8]$, & $<[0.4,0.5]$, & $<[0.5,0.6]$, \\
& {$[0.3,0.4]>$} & {$[0.1,0.3]>$} & {$[0.1,0.2]>$} & {$[0.2,0.3]>$} & {$[0.3,0.4]>$} \\
\hline \multirow{2}{*}{$A_{4}$} & $<[0.3,0.4]$, & $<[0.1,0.3]$, & $<[0.1,0.2]$, & $<[0.2,0.3]$, & $<[0.3,0.4]$, \\
& {$[0.4,0.5]>$} & {$[0.6,0.7]>$} & {$[0.5,0.8]>$} & {$[0.4,0.5]>$} & {$[0.5,0.6]>$} \\
\hline
\end{tabular}

In the following, we will solve this problem with the proposed decision making method, and the calculation steps are as follows:

Step 1. Establish a comprehensive IVIF decision matrix using the IIFHG operator; see Table 5.

Table 5. Comprehensive decision matrix $D$.

\begin{tabular}{ccccccc}
\hline \multirow{2}{*}{ Alternatives } & \multicolumn{5}{c}{ Attributes } \\
\cline { 2 - 6 } & $o_{1}$ & $o_{2}$ & $o_{3}$ & $o_{4}$ & $o_{5}$ \\
\hline \multirow{2}{*}{$A_{1}$} & $<[0.4385,0.6199]$, & $<[0.3000,0.4573]$, & $<[0.6116,0.7117]$, & $<[0.5000,0.6395]$, & $<[0.1323,0.3623]$, \\
& {$[0.1600,0.2910]>$} & {$[0.3481,0.4814]>$} & {$[0.1195,0.2196]>$} & {$[0.1000,0.2602]>$} & {$[0.3870,0.5548]>$} \\
\hline \multirow{2}{*}{$A_{2}$} & $<[0.3502,0.4797]$, & $<[0.1138,0.3010]$, & $<[0.3379,0.4387]$, & $<[0.1758,0.3134]$, & $<[0.6395,0.7521]$, \\
& {$[0.3241,0.4827]>$} & {$[0.2691,0.5118]>$} & {$[0.3918,0.4930]>$} & {$[0.5627,0.6752]>$} & {$[0.1195,0.2196]>$} \\
\hline \multirow{2}{*}{$A_{3}$} & $<[0.3516,0.4906]$, & $<[0.6395,0.7711]$, & $<[0.5213,0.7804]$, & $<[0.4387,0.6252]$, & $<[0.5392,0.6759]$, \\
& {$[0.3000,0.4436]>$} & {$[0.1000,0.2289]>$} & {$[0.1000,0.2196]>$} & {$[0.2289,0.3292]>$} & {$[0.1295,0.3045]>$} \\
\hline \multirow{2}{*}{$A_{4}$} & $<[0.3000,0.4170]$, & $<[0.1000,0.2103]$, & $<[0.1000,0.2366]$, & $<[0.2103,0.3109]$, & $<[0.1849,0.3121]$, \\
& {$[0.3241,0.4930]>$} & {$[0.6208,0.7897]>$} & {$[0.5658,0.7634]>$} & {$[0.4200,0.5968]>$} & {$[0.5203,0.6484]>$} \\
\hline
\end{tabular}

Step 2. Calculate the attribute weights according to Equation (14). Set $p=0.1$, and then we can establish the following programming model: 


$$
\begin{aligned}
& \max S=2.1064 w_{1}+1.8329 w_{2}+2.1551 w_{3}+1.9530 w_{4}+2.1148 w_{5} \\
& \text { s.t. }\left\{\begin{array}{l}
w_{1} \leq 0.3 \\
0.1 \leq w_{2} \leq 0.2 \\
0.2 \leq w_{3} \leq 0.5 \\
w_{5} \leq 0.4 \\
0.1 \leq w_{4} \leq 0.3 \\
w_{3}-w_{2} \geq w_{5}-w_{4} \\
w_{4} \geq w_{1} \\
w_{3}-w_{1} \leq 0.1 \\
w_{1}+w_{2}+w_{3}=1
\end{array}\right.
\end{aligned}
$$

We use Matlab software to solve this model, and get the optimum attribute weight vector

$$
\boldsymbol{W}=(0.16,0.10,0.26,0.16,0.32)^{T}
$$

Step 3. The PIS $\left(A^{+}\right)$is defined as:

$$
A^{*}=\left(\tilde{a}_{1}^{+}, \tilde{a}_{2}^{+}, \ldots, \tilde{a}_{5}^{+}\right)=(<[1,1],[0.0]>,<[1,1],[0.0]>, \ldots,<[1,1],[0.0]>)
$$

Step 4. According to Equation (15), the similarity measures $S\left(A_{i}\right)$ of each alternative from PIS are calculated as:

$$
S\left(A_{1}\right)=0.5645, S\left(A_{2}\right)=0.5246, S\left(A_{3}\right)=0.6650 \text { and } S\left(A_{4}\right)=0.3157
$$

Step 5. According the value of $S\left(A_{i}\right)(i=1,2,3)$, the ranking order of all alternatives is $A_{3} \succ A_{1} \succ A_{2} \succ A_{4}$, and $A_{3}$ is the desirable alternative. This result is in agreement with the one obtained in Park et al. [27]. The attitude factor $p$ reflects the attitude of the decision maker to recognize the interval number, and it is more consistent with the objective reality.

\section{Conclusions}

In this paper, the similarity measure of IVIF sets is analyzed and studied. Firstly, we construct a new similarity measure, and then based on this similarity, a new decision-making method is put forward for an MADM problem with attribute values expressed by IVIF numbers and partially known information on attribute weights. The advantages of the proposed decision-making method are as follows:

(1) Similarity measure can be used to measure the similarity between sets, and it can be used to avoid the deficiency of IVIF additive operation to some extent;

(2) A new method for determining the weights of attributes is proposed by establishing the maximum similarity optimization model. An example of supplier selection showed the proposed method is feasible and effective;

(3) The proposed similarity measure can be applied to pattern recognition, medical diagnosis and cluster analysis fields, and the proposed MADM method can be applied to such issues as investment project selection, site selection, and emergency management and decisions. 


\section{Acknowledgments}

This paper was supported by the National Natural Science Foundation of China (No. 11461029) and Natural Science Foundation of Jiangxi Province (No. 20132BAB211015 and No. 2014BAB201009). Science and Technology Research Project of Jiangxi Provincial Education Department (No. GJJ14449), and Natural Science Foundation of Jiangxi University of Science and Technology (JXUST) (No. NSFJ2014-G38). The authors would like to sincerely thank the editor and reviewers for their valuable comments and suggestions.

\section{Author Contributions}

Haiping Ren developed the idea for the article. He developed and wrote the manuscript. Guofu Wang constructed the optimization model for determining attribute weight. Both authors have read and approved the final manuscript.

\section{Conflicts of Interest}

The authors have declared that no conflicts of interest exists.

\section{References}

1. Kulak, O; Goren, H.G.; Supciller, A.A. A new multi criteria decision making approach for medical imaging systems considering risk factors. Appl. Soft Comput. 2015, 35, 931-941.

2. Zhu, B.; Xu, Z.S. A fuzzy linear programming method for group decision making with additive reciprocal fuzzy preference relations. Fuzzy Sets Syst. 2014, 246, 19-33.

3. Merigó, J.M.; Casanovas, M.; Liu, P.D. Decision making with fuzzy induced heavy ordered weighted averaging operators. Int. J. Fuzzy Syst. 2014, 16, 277-289.

4. Zadeh, L.A. Fuzzy sets. Inf. Control 1965, 18, 338-356.

5. Jahan, A.; Edwards, K.L. VIKOR method for material selection problems with interval numbers and target-based criteria. Mater. Des. 2013, 47, 759-765.

6. Chang, T.H. Fuzzy VIKOR method: A case study of the hospital service evaluation in Taiwan. Inf. Sci. 2014, 271, 196-212.

7. Atanassov, K.T. Intuitionistic Fuzzy Sets. Fuzzy Sets Syst. 1986, 20, 87-96.

8. Atanassov, K.T.; Gargov, G. Interval-valued intuitionistic fuzzy sets. Fuzzy Sets Syst. 1989, 31, 343-349.

9. Chen, T.Y.; Li, C.H. Objective weights with intuitionistic fuzzy entropy measures and computational experiment analysis. Appl. Soft Comput. 2011, 11, 5411-5423.

10. Su, Z.X.; Chen, M.Y.; Xia, G.P.; Wang, L. An interactive method for dynamic intuitionistic fuzzy multi-attribute group decision making. Expert Syst. Appl. 2011, 38, 15286-15295.

11. Wang, Z.J.; Li, K.W.; Xu, J.H. A mathematical programming approach to multi-attribute decision making with interval-valued intuitionistic fuzzy assessment information. Expert Syst. Appl. 2011, $38,12462-12469$. 
12. De Miguel, L.; Bustince, H.; Fernandez, J.; Induráin, E.; Kolesárová, A.; Mesiar, R. Construction of admissible linear orders for interval-valued Atanassov intuitionistic fuzzy sets with an application to decision making. Inf. Fusion 2016, 27, 189-197.

13. Yu, D.J.; Merigó, J.M.; Zhou, L.G. Interval-valued multiplicative intuitionistic fuzzy preference relations. Int. J. Fuzzy Syst. 2013, 15, 412-422.

14. Liao, H.C.; Xu, Z.S.; Zeng, X.J.; Merigó, J.M. Framework of group decision making with intuitionistic fuzzy preference information. IEEE Trans. Fuzzy Syst. 2015, 23, 1211-1227.

15. Li, D.F.; Chen, C.T. New similarity measures of intuitionistic fuzzy sets and application to pattern recognition. Pattern Recogn. Lett. 2002, 23, 221-225.

16. Mitchell, H.B. On the Dengfeng-Chuntian similarity measure and its application to pattern recognition. Pattern Recogn. Lett. 2003, 24, 3101-3104.

17. Baccour, L.; Alimi, A.M.; John, R.I. Similarity measures for intuitionistic fuzzy sets: State of the art. J. Intell. Fuzzy Syst. 2013, 24, 37-49.

18. Hwang, C.M.; Yang, M.S. New construction for similarity measures between intuitionistic fuzzy sets based on lower, upper and middle fuzzy sets. Int. J. Fuzzy Syst. 2013, 15, 371-378.

19. Xu, Z.S. Intuitionistic fuzzy hierarchical clustering algorithms. J. Syst. Eng. Electron. 2009, 20, 90-97.

20. Xu, Z.S.; Chen, J. On geometric aggregation over interval-valued intuitionistic fuzzy information. In Proceedings of the Fourth International Conference on Fuzzy Systems and Knowledge Discovery (FSKD2007), Haikou, China, 24-27 August 2007; Volume 2, pp. 466-471.

21. Zhang, Q.S.; Jiang, S.Y.; Jia, B.G.; Luo, S.H. Some information measures for interval-valued intuitionistic fuzzy sets. Inf. Sci. 2010, 180, 5130-5145.

22. Xu, Z.S. Multi-person multi-attribute decision making models under intuitionistic fuzzy environment. Fuzzy Optim. Decis. Mak. 2007, 6, 221-236.

23. Chen, S.M.; Tan, J.M. Handing multicriteria fuzzy decision-making problems based vague set theory. Fuzzy Sets Syst. 1994, 67, 163-172.

24. Wu, J.Z.; Zhang, Q. Multicriteria decision making method based on intuitionistic fuzzy weighted entropy. Expert Syst. Appl. 2011, 38, 916-922.

25. Wang, J.Q.; Wang, P. Intuitionistic linguistic fuzzy multi-criteria decision-making method based on intuitionistic fuzzy entropy. Control Decis. 2012, 27, 1694-1698.

26. Chen, Z.P.; Yang, W. A new multiple attribute group decision making method in intuitionistic fuzzy setting. Appl. Math. Model. 2011, 35, 4424-4437.

27. Park, D.G.; Kwun, Y.C.; Park, J.H.; Park, I.Y. Correlation coefficient of interval-valued intuitionistic fuzzy sets and its application to multiple attribute group decision making problems. Math. Comput. Model. 2009, 50, 1279-1293.

(C) 2015 by the authors; licensee MDPI, Basel, Switzerland. This article is an open access article distributed under the terms and conditions of the Creative Commons Attribution license (http://creativecommons.org/licenses/by/4.0/). 